\title{
Wolff's inequality in multi-parameter Morrey spaces
}

\author{
Tord Sjödin, Umeå
}

April 20, 2011

\begin{abstract}
We prove Wolff inequalities for multi-parameter Riesz potentials and Wolff potentials in Lebesque spaces $L^{p}\left(R^{d}\right)$ and multi-parameter Morrey spaces $L_{\lambda}^{p}\left(R^{d}\right)$, where $R^{d}=R^{n_{1}} \times R^{n_{2}} \times \cdots \times R^{n_{k}}, \lambda=$ $\left(\lambda_{1}, \ldots, \lambda_{k}\right)$ and $0<\lambda_{i} \leq n_{i}, 1 \leq i \leq k$, in the dyadic case as well as in the non-dyadic (continuous) case.
\end{abstract}

Key words and phrases: Riesz potential, Wolff potential, multi-parameter potential, dyadic potential, Wolff's inequality, Morrey spaces

AMS 2000 Subject Classsification: Primary 15 A 03, Secondary 15 A 63, $46 \mathrm{C} 05$

Address: Department of Mathematics and Mathematical Statsitics, University of Umeå, 90187 Umeå, Sweden

\section{Introduction.}

The nonlinear potential theory for Riesz potentials and more general potentials of functions in the Lebesque class $L^{p}\left(R^{d}\right)$ is well known, see [1] and [10] and the references contained there. A major step in this theory was the so called Wolff's inequality [4] and its applications to various parts of the theory. The corresponding theory for functions in the Morrey spaces $L_{\lambda}^{p}\left(R^{d}\right)$, with its roots in [3], [5], [7] and [9], is more recent and a part of it was developed in [2]. In this paper we consider the extension of two basic inequalities in this theory to a multi-parameter setting, see [8]. Our main result (Theorem 2) is a generalization of Wolff's inequality, refered to above, 
to the multi-parameter Morrey spaces $L_{\lambda}^{p}\left(R^{d}\right)$.

We consider multi-parameter Riesz potentials and Wolff potentials in an Euclidean space $R^{d}=R^{n_{1}} \times R^{n_{2}} \cdots \times R^{n_{k}}$, where $d=n_{1}+n_{2}+\cdots+n_{k}$ and each $R^{n_{i}}$ is a standard Euclidean space. The multi-parameter Riesz potential $R_{\rho} \mu$ of a nonnegative measure $\mu$ in $R^{d}$ is defined by

$$
R_{\rho} \mu(\mathbf{x})=\int\left|x_{1}-y_{1}\right|^{\rho_{1}-n_{1}} \cdots\left|x_{k}-y_{k}\right|^{\rho_{k}-n_{k}} d \mu\left(y_{1}, \ldots, y_{k}\right),
$$

where $\mathbf{x}=\left(x_{1}, \ldots, x_{k}\right)$. Here $\rho=\left(\rho_{1}, \ldots, \rho_{k}\right)$ and $0<\rho_{i}<n_{i}, 1 \leq i \leq k$. The case $k=1$ gives the classical Riesz potential in $R^{d}$, see [1]. We are mainly going to work with the dyadic counterpart $R_{\rho}^{d y} \mu$ of $R_{\rho} \mu$, defined in (1) below and comment on the non-dyadic (continuous) case at the end of Section 3 .

We also define the corresponding dyadic multi-parameter Wolff potential $W_{\rho, p}^{d y} \mu$ in (2) and prove Wolff's inequality in this setting (Theorem 1). The case $k=1$ was proved by Th. Wolff in [4], while the present author settled the case $k=2$ in [8]. D. R. Adams and Jie Xiao [2] studied the potential theory for the Riesz kernel in the Morrey space $L_{\lambda}^{p}\left(R^{d}\right)$ and proved a Wolff inequality. We define the dyadic multi-parameter Morrey-Wolff potentials $W_{\rho, \lambda, p}^{d y} \mu$ and prove the corresponding Wolff inequality (Theorem 2) in the multi-parameter Morrey spaces $L_{\lambda}^{p}\left(R^{d}\right)$.

The plan of this paper is as follows. Section 2 contains our notation and definitions, our results are stated in Section 3 and proved in Section 4.

\section{Preliminaries.}

We work in Euclidean spaces $R^{d}=R^{n_{1}} \times R^{n_{2}} \times \cdots R^{n_{k}}$, where $d=n_{1}+$ $n_{2}+\cdots n_{k}$ and each $R^{n_{i}}$ is a standard Euclidean space. Points in $R^{d}$ are written $\mathbf{x}=\left(x_{1}, x_{2}, \ldots, x_{k}\right)$, where $x_{i} \in R^{n_{i}}, 1 \leq i \leq k$. Our basic geometric object is a dyadic rectangle $I=I_{1} \times I_{2} \times \cdots \times I_{k}$, where each $I_{i}$ is a standard dyadic cube in $R^{n_{i}}, 1 \leq i \leq k$. The side length of a cube $I$ in $R^{m}$ is denoted by $l(I)$ and $|I|=l(I)^{m}$ is the $m$-dimensional Lebesque measure of $I$. If $\mathbf{a}=\left(a_{1}, a_{2}, \ldots, a_{k}\right)$, where $a_{i}>0$ for $1 \leq i \leq k$, we define a multi-parameter ball $B(\mathbf{x} ; \mathbf{a})$ as the Cartesian product of standard balls $B\left(x_{i}, a_{i}\right)$ in $R^{n_{i}}, 1 \leq i \leq k$. We let $M_{+}\left(R^{d}\right)$ denote the class of nonnegative Borel measures in $R^{d}$, finite on compact sets. The Lebesque measure of a set $E \subset R^{d}$ is denoted by $|E|$ and Lebesque integration is written $\int d x$. 
The standard Lebesque space of $p$-integrable functions is denoted by $L^{p}\left(R^{d}\right)$

We use the following vector notation to simplify our presentation. A $k$-vector is a vector $\alpha=\left(\alpha_{1}, \ldots, \alpha_{k}\right)$, with real numbers $\alpha_{i}, 1 \leq i \leq k$. We say that $\alpha>0$, if all $\alpha_{i}>0$. If $\beta$ is another $k$-vector we say that $\alpha>\beta$ if $\alpha-\beta>0$. When $\alpha>0$ and $\beta$ are $k$-vectors we define $\alpha^{\beta}=\alpha_{1}^{\beta_{k}} \cdot \alpha_{2}^{\beta_{2}} \cdots \alpha_{k}^{\beta_{k}}$. Let $I=I_{1} \times \cdots \times I_{k}$ be a dyadic rectangle in $R^{d}=R^{n_{1}} \times \cdots \times R^{n_{k}}$ and define $k$-vectors $l(I)=\left(l\left(I_{1}\right), \ldots, l\left(I_{k}\right)\right)$ and $n=\left(n_{1}, \ldots, n_{k}\right)$. Then $l(I)^{\alpha}=l\left(I_{1}\right)^{\alpha_{1}} \cdot l\left(I_{2}\right)^{\alpha_{2}} \cdots l\left(I_{k}\right)^{\alpha_{k}}$, for any $k$-vector $\alpha$.

We can now define the dyadic multi-parameter Riesz potential $R_{\rho}^{d y} \mu$ of the measure $\mu \in M_{+}\left(R^{d}\right)$ using this notation by

$$
R_{\rho}^{d y} \mu(\mathbf{x})=\sum_{I} l(I)^{\rho-n} \cdot \mu(I) \cdot \chi_{I}(\mathbf{x})
$$

and the dyadic multi-parameter Wolff potential by

$$
W_{\rho, p}^{d y} \mu(\mathbf{x})=\sum_{I} l(I)^{p(\rho-n)+n} \cdot \mu(I)^{p-1} \chi_{I}(\mathbf{x})
$$

where in both cases the sum is over all dyadic rectangles $I=I_{1} \times I_{2} \times \cdots \times I_{k}$ in $R^{d}$.

We study the dyadic multi-parameter Riesz potentials and Wolff potentials in the Lebesque spaces $L^{p}\left(R^{d}\right)$ and more generally in the multi-parameter Morrey spaces $L_{\lambda}^{p}\left(R^{d}\right)$ defined as follows.

Definition 1 Let $1<p<\infty, \lambda=\left(\lambda_{1}, \ldots, \lambda_{k}\right)$ and $0<\lambda \leq n$. A function $f$ belongs to $L_{\lambda}^{p}\left(R^{d}\right)$ if

$$
\|f\|_{p, \lambda}^{p}=\sup _{I} l(I)^{\lambda-n} \int_{I}|f|^{p} d x
$$

is finite, where supremum is over all dyadic rectangles $I$ in $R^{d}$.

The spaces $L_{\lambda}^{p}\left(R^{d}\right)$ for $k=1$ go back to C. B. Morrey in the 1930's and were further studied by Stampacchia [9] and S Campanato [3]. See [5] and [7] and the references contained there. For applications to partial equations, see [6]. Note that when $\lambda_{i}=n_{i}$, for all $1 \leq i \leq k$, we get $L_{\lambda}^{p}\left(R^{d}\right)=L^{p}\left(R^{d}\right)$.

We define the corresponding multi-parameter Wolff potentials as follows. 
Let $1<p<\infty$ and let $0<\rho<n, 0<\lambda \leq n$ be as above, then the dyadic Morrey-Wolff potential $W_{\rho, \lambda, p}^{d y} \mu$ of a measure $\mu \in M_{+}\left(R^{d}\right)$ is defined by

$$
W_{\rho, \lambda, p}^{d y} \mu(\mathbf{x})=\sum_{I} l(I)^{p(\rho-n)+\lambda} \cdot \mu(I)^{p-1} \chi_{I}(\mathbf{x})
$$

where the sum is over all dyadic rectangles $I$ in $R^{d}$.

\section{Main results.}

The following multi-parameter Wolff Inequality in $L^{p}\left(R^{d}\right)$ is our first result.

Theorem 1 Let $1<p<\infty, 1 / p+1 / q=1,0<\rho<n / q$ and let $\mu \in$ $M_{+}\left(R^{d}\right)$. Then

$$
\int W_{\rho, p}^{d y} \mu(\mathbf{x}) d \mu(\mathbf{x}) \leq \int\left[R_{\rho}^{d y} \mu(\mathbf{x})\right]^{p} d \mathbf{x} \leq C \cdot \int W_{\rho, p}^{d y} \mu(\mathbf{x}) d \mu(\mathbf{x}),
$$

where the constant $C$ only depends on $d, p$ and $\rho$.

Theorem 1 is a special case of the next theorem, which is our multi-parameter Wolff Inequality in the Morrey space $L_{\lambda}^{p}\left(R^{d}\right)$ and is our main result.

Theorem 2 Let $1<p<\infty, 1 / p+1 / q=1, \rho, d$ and $\lambda$ be as above, $0<$ $\rho<n / q, 0<\lambda \leq n$ and let $\mu \in M_{+}\left(R^{d}\right)$. Then

$$
\left\|R_{\rho}^{d y} \mu\right\|_{p, \lambda}^{p} \leq C \cdot \int W_{\rho, \lambda, p}^{d y} \mu(\mathbf{x}) d \mu(\mathbf{x}),
$$

where $C$ only depends on $d, p, \rho$ and $\lambda$.

In the non-dyadic (continuous) case we define a multi-parameter Wolff potential by

$$
W_{\rho, p} \mu(\mathbf{x})=\int_{0}^{\infty} \ldots \int_{0}^{\infty} \mathbf{a}^{p(\rho-n)} \cdot \mu B(\mathbf{x} ; \mathbf{a})^{p-1} \frac{d \mathbf{a}}{\mathbf{a}},
$$

where $\mathbf{a}=\left(a_{1}, a_{2}, \ldots, a_{k}\right)$ and $\frac{d \mathbf{a}}{\mathbf{a}}=\frac{d a_{1}}{a_{1}} \frac{d a_{2}}{a_{2}} \ldots \frac{d a_{k}}{a_{k}}$. Then Theorem 1 holds, modulo a multiplicative constant depending on d, p and $\rho$, with $R_{\rho}^{d y} \mu$ and $W_{\rho, p}^{d y} \mu$ replaced by $R_{\rho} \mu$ and $W_{\rho, p} \mu$ respectively. The main point is that

$$
R_{\rho} \mu(\mathbf{x}) \leq \widetilde{R}_{\rho}^{d y} \mu(\mathbf{x})=C \cdot \sum_{I} l(I)^{\rho-d} \cdot \mu(\widetilde{I}) \cdot \chi_{I}(\mathbf{x}),
$$


where $\widetilde{I}$ denotes the rectangle concentric to $I$ with $l(\widetilde{I})=3 \cdot l(I)$. Then $(3)$ remains true by inequality $(\star)$ in [4], p. 170 and the result follows from standard estimates. An analogous argument proves Theorem 2 in the nondyadic case with $R_{\rho}^{d y} \mu$ and $W_{\rho, \lambda, p}^{d y}$ replaced by $R_{\rho} \mu$ and $W_{\rho, \lambda, p}$ respectively, where

$$
W_{\rho, \lambda, p} \mu(\mathbf{x})=\int_{0}^{\infty} \ldots \int_{0}^{\infty} \mathbf{a}^{p(\rho-n)+\lambda} \cdot \mu B(\mathbf{x} ; \mathbf{a})^{p-1} \frac{d \mathbf{a}}{\mathbf{a}},
$$

denotes the corresponding non-dyadic Morrey-Wolff potential.

\section{Proofs.}

For the readers convenience and for the clearence of the exposition we give a proof of the special case in Theorem 1 before we prove the more complicated Theorem 2.

Proof of Theorem 1. The first inequality follows from the elementary inequality $\left(\sum_{i} a_{i}\right)^{p} \geq \sum_{i} a_{i}^{p}$, for any $a_{i} \geq 0$ and $p \geq 1$. To prove the second inequality we first assume that the support of $\mu$ is contained in a fixed dyadic rectangle $\bar{I}=\bar{I}_{1} \times \cdots \times \bar{I}_{k}$ in $R^{d}$. We claim that then

$$
\int_{\bar{I}}\left(\sum_{I} l(I)^{\rho-n} \cdot \mu(I) \cdot \chi_{I}(\mathbf{x})\right)^{p} d \mathbf{x} \leq C \cdot \sum_{I \subset \bar{I}} l(I)^{p(\rho-n)} \cdot l(I)^{n} \cdot \mu(I)^{p},
$$

where both sums are over all dyadic rectangles $I=I_{1} \times \cdots \times I_{k}$ and $C$ only depends on $d, p$ and $\rho$. It is clearly no loss of generality to assume that $I \subset \bar{I}$ in the sum on the left hand side of (3). In the case $k=1,(3)$ was proved in $[4]$, p. 170 , for $l(\bar{I})=1$. The case of a general $\bar{I}$ follows by homogeneity. The case $k=2$ was proved by the author in [8], Theorem 4.2. The case of a general $k$ is now proved by induction over the number $k$ of factors.

Assume that (3) holds for $k$ factors, where $k \geq 1$. We keep the space $R^{d}$ above and consider $R^{d} \times R^{m}$, for some Euclidean space $R^{m}$, where points are denoted by $(\mathbf{x}, \mathbf{s}), \mathbf{x} \in R^{d}$ and $\mathbf{s} \in R^{m}$. Let $\bar{J}$ be a cube in $R^{m}, 0<\rho_{m}<m$ and let $\mu \in M_{+}\left(R^{d} \times R^{m}\right)$ be a measure with support in $\bar{I} \times \bar{J}$. For a fixed point $s$ in $\bar{J}$ we define a measure $\nu_{\mathbf{s}}$ in $R^{d}$, supported in $\bar{I}$, by

$$
\nu_{\mathbf{s}}(E)=\sum_{J \subset \bar{J}} l(J)^{\rho_{m}-m} \cdot \mu(E \times J) \cdot \chi_{J}(\mathbf{s}) .
$$


Then

$$
\int_{\bar{I}}\left(\sum_{I} l(I)^{\rho-n} \cdot \nu_{\mathbf{s}}(I) \cdot \chi_{I}(\mathbf{x})\right)^{p} d \mathbf{x} \leq C \cdot \sum_{I \subset \bar{I}} l(I)^{p(\rho-n)} \cdot l(I)^{n} \cdot \nu_{\mathbf{s}}(I)^{p}
$$

by our induction hypothesis applied to the measure $\nu_{\mathbf{s}}$ and

$$
\int_{\bar{J}} \nu_{\mathbf{s}}(I)^{p} d \mathbf{s} \leq C \cdot \sum_{J \subset \bar{J}} l(J)^{p\left(\rho_{m}-m\right)} \cdot l(J)^{m} \cdot \mu(I \times J)^{p}
$$

by the case $k=1$ for the measure $\mu$. Combining (4) and (5) proves (3) for $(k+1)$ factors and settles our claim.

To complete the proof of Theorem 1 we take $\mu \in M_{+}\left(R^{d}\right)$ and let $\mu_{M}$ be the restriction of $\mu$ to the dyadic cube $I_{M}=\left[0,2^{M}\right]^{d}$, for a positive integer $M$. Then

$$
\int_{I_{M}}\left[R_{\rho}^{d y} \mu_{M}(\mathbf{x})\right]^{p} d \mu(\mathbf{x}) \leq C \cdot \int W_{\rho, \lambda, p}^{d y} \mu(\mathbf{x}) d \mu_{M}(\mathbf{x}) \leq C \cdot \int W_{\rho, \lambda, p}^{d y} \mu(\mathbf{x}) d \mu(\mathbf{x})
$$

by (3) and letting $M \rightarrow \infty$ gives

$$
\int_{D}\left[R_{\rho}^{d y} \mu(\mathbf{x})\right]^{p} d \mu(\mathbf{x}) \leq C \cdot \int W_{\rho, \lambda, p}^{d y} \mu(\mathbf{x}) d \mu(\mathbf{x})
$$

by Monotone Convergence, where $D$ is the quadrant $[0, \infty)^{d}$. Repeating this argument for the other quadrants proves Theorem 1.

Our proof of Theorem 2 uses the result in Theorem 1 in an essential way and also contains a much simplified proof of the case $k=1$ in [2], p. 1656.

Proof of Theorem 2. Let $\bar{I}=\bar{I}_{1} \times \cdots \times \bar{I}_{k}$ be a fixed dyadic rectangle in $R^{d}$. We must prove that

$$
\int_{\bar{I}}\left[R_{\rho}^{d y} \mu(\mathbf{x})\right]^{p} d \mu(\mathbf{x}) \leq C \cdot l(\bar{I})^{n-\lambda} \cdot \int W_{\rho, \lambda, p}^{d y} \mu(\mathbf{x}) d \mu(\mathbf{x}),
$$

for some constant $C$ that only depends on $d, \rho, \lambda$ and $p$. We split $\mu$ in three terms as follows. Let $\mu=\mu_{1}+\mu_{2}+\mu_{3}$, where $\mu_{1}$ and $\mu_{2}$ are the restrictions of $\mu$ to $\bar{I}$ and $\bar{I}_{1}^{c} \times \cdots \times \bar{I}_{k}^{c}$ respectively. We start with the principal term $\mu_{1}$. Then

$$
\int_{\bar{I}}\left[R_{\rho}^{d y} \mu_{1}(\mathbf{x})\right]^{p} d \mu(\mathbf{x}) \leq C \int_{\bar{I}}\left(\sum_{I \subset \bar{I}} l(I)^{\rho-n} \cdot \mu_{1}(I) \cdot \chi_{I}(\mathbf{x})\right)^{p} d \mathbf{x} \leq
$$




$$
\begin{gathered}
\leq C \cdot \sum_{I \subset \bar{I}} l(I)^{p(\rho-n)} \\
\cdot l(I)^{n} \cdot \mu(I)^{p}=C \cdot l(\bar{I})^{n-\lambda} \cdot \sum_{I \subset \bar{I}}\left(l(I)^{\rho-n+\lambda / p} \cdot \mu(I)\right)^{p} \leq \\
\leq C \cdot l(\bar{I})^{n-\lambda} \cdot \int W_{\rho, \lambda, p}^{d y} \mu(\mathbf{x}) d \mu(\mathbf{x}),
\end{gathered}
$$

by (3) and the definition of $W_{\rho, \lambda, p}^{d y} \mu(\mathbf{x})$. This proves (6) for the principal term $\mu_{1}$.

Next we consider the second and easiest term $\mu_{2}$. We show that $R_{\rho}^{d y} \mu_{2}(\mathbf{x})$ is bounded on $\bar{I}$. For each $i, 1 \leq i \leq k$, we let $\bar{I}_{i}^{j_{i}}$ denote the unique dyadic cube containing $\bar{I}_{i}$ with $l\left(\bar{I}_{i}^{j_{i}}\right)=2^{j_{i}} \cdot l\left(\bar{I}_{i}\right), j_{i} \geq 0$. Define a $k$-vector $j=\left(j_{1}, j_{2}, \ldots, j_{k}\right)$ and put $\bar{I}^{j}=\bar{I}_{1}^{j_{1}} \times \bar{I}_{2}^{j_{2}} \times \cdots \times \bar{I}_{k}^{j_{k}}$. Then for any $x \in \bar{I}$

$$
R_{\rho}^{d y} \mu_{2}(\mathbf{x})=\sum^{\prime} l(I)^{\rho-n} \cdot \mu(I) \cdot \chi_{I}(x)+\sum_{j \geq 0} l\left(\bar{I}^{j}\right)^{\rho-n} \cdot \mu_{2}\left(\bar{I}^{j}\right),
$$

where the first sum $\sum^{\prime}$ is over all dyadic rectangles $I=I_{1} \times I_{2} \times \cdots \times I_{k}$ with $I_{j} \subset \bar{I}_{j}$ for some $1 \leq j \leq k$ and consequently equals zero. Hence we get the upper bound

$$
\begin{gathered}
R_{\rho}^{d y} \mu_{2}(\mathbf{x}) \leq \sum_{j \geq 0} l\left(\bar{I}^{j}\right)^{\rho-n} \cdot \mu_{2}\left(\bar{I}^{j}\right)=\sum_{j \geq 0} l\left(\bar{I}^{j}\right)^{-\lambda / p} \cdot l\left(\bar{I}^{j}\right)^{\rho-n+\lambda / p} \mu_{2}\left(\bar{I}^{j}\right) \leq \\
\leq\left(\sum_{j \geq 0} l\left(\bar{I}^{j}\right)^{-\lambda q / p}\right)^{1 / q} \cdot\left(\sum_{j \geq 0} l\left(\bar{I}^{j}\right)^{(\rho-n) p+\lambda} \cdot \mu_{2}\left(\bar{I}^{j}\right)^{p}\right)^{1 / p} \leq \\
\leq C \cdot l(\bar{I})^{-\lambda / p} \cdot\left(\sum_{j \geq 0} l\left(\bar{I}^{j}\right)^{(\rho-n) p+\lambda} \cdot \mu_{2}\left(\bar{I}^{j}\right)^{p}\right)^{1 / p},
\end{gathered}
$$

by Hölder's inequality, with $1 / p+1 / q=1$. Integrating to the $p$-th power over $\bar{I}$ gives that

$$
\int_{\bar{I}}\left[R_{\rho} \mu_{2}(\mathbf{x})\right]^{p} d \mathbf{x} \leq C \cdot l(\bar{I})^{n-\lambda} \cdot \int_{\bar{I}} W_{\rho, \lambda, p}^{d y} \mu(\mathbf{x}) d \mu(\mathbf{x})
$$

by the definition of $W_{\rho, \lambda, p}^{d y} \mu(\mathbf{x})$, which proves (6) for $\mu_{2}$.

Before we proceed to the last term we need some more notation. If $A=$ $\left\{a_{1}, a_{2}, \cdots, a_{l}\right\}, a_{1}<a_{2}<\cdots<a_{l}$, is a nonempty subset of $\{1,2, \ldots, k\}$ and $v=\left(v_{1}, v_{2}, \ldots, v_{k}\right)$ is a $k$-vector, we define the $l$-vector $v_{A}=\left(v_{a_{1}}, v_{a_{2}}, \ldots, v_{a_{l}}\right)$. 
If $v>0$ and $\alpha$ is any other $k$-vector we put $v_{A}^{\alpha}=\left(v_{A}\right)^{\alpha_{A}}$, as defined above. In particular, if $\mathbf{x}=\left(x_{1}, x_{2}, \ldots, x_{k}\right)$ is in $R^{d}$ then $\mathbf{x}_{A}$ is a point in $R^{A}=R^{n_{a_{1}}} \times R^{n_{a_{2}}} \times \cdots \times R^{n_{a_{l}}}$ and if $I$ is a dyadic rectangle we put $I_{A}=I_{a_{1}} \times I_{a_{2}} \times \cdots \times I_{a_{l}}$.

Now we consider the last term $\mu_{3}$, which is a mixture of $\mu_{1}$ and $\mu_{2}$ in a sense to be described below. We split $\{1,2, \ldots, k\}$ into two nonempty and disjoint sets $A=\left\{a_{1}, a_{2}, \ldots, a_{l}\right\}$ and $B=\{1,2, \ldots, k\} \backslash A$ and consider the class of dyadic rectangles $I=I_{1} \times I_{2} \times \cdots \times I_{k}$ such that $I_{i} \subset \bar{I}_{i}$, for $i \in A$, and $2 \bar{I}_{i} \subset I_{i}$, for $i \in B$. Let $\mathbf{x} \in \bar{I}$ then $R_{\rho}^{d y} \mu_{3}(\mathbf{x})$ only depends on $\mathbf{x}_{A}$ and we have

$$
\begin{gathered}
R_{\rho}^{d y} \mu_{3}(\mathbf{x})=R_{\rho}^{d y} \mu_{3}\left(\mathbf{x}_{A}\right) \leq \\
\leq C \cdot \sum_{I_{i} \subset \bar{I}_{i}, i \in A}\left(l(I)^{\rho-n}\right)_{A} \cdot\left(\sum_{\bar{I}_{i} \subset I_{i}, i \in B}\left(l(I)^{\rho-n}\right)_{B} \cdot \mu(I)\right) \cdot \chi_{I_{A}}\left(\mathbf{x}_{A}\right),
\end{gathered}
$$

where

$$
\nu\left(I_{A}\right)=\sum_{\bar{I}_{i} \subset I_{i}, i \in B} l(I)_{B}^{\rho-n} \cdot \mu(I)
$$

defines $\nu$ on dyadic rectangles $I_{A}$ and can be extended to a nonnegative measure in $R^{A}$ in the standard way. We now get

$$
\begin{gathered}
\int_{\bar{I}}\left[R_{\rho}^{d y} \mu_{3}(\mathbf{x})\right]^{p} d \mathbf{x}=l(\bar{I})_{B}^{n} \cdot \int_{\bar{I}_{A}}\left[R_{\rho}^{d y} \mu_{3}\left(\mathbf{x}_{A}\right)\right]^{p} d \mathbf{x}_{A} \leq \\
\leq C \cdot l(\bar{I})_{B}^{n} \cdot l(\bar{I})_{A}^{n-\lambda} \cdot \sum_{I_{i} \subset \bar{I}_{i}, i \in A}\left(l\left(I_{A}\right)_{A}^{(\rho-n)+\lambda / p} \cdot \nu\left(I_{A}\right)\right)^{p}
\end{gathered}
$$

by the first case, with $\mu$ replaced by $\nu$, and

$\nu\left(I_{A}\right)^{p}=\left(\sum_{\bar{I}_{i} \subset I_{i}, i \in B} l(I)_{B}^{\rho-n} \cdot \mu(I)\right)^{p} \leq C \cdot l(\bar{I})_{B}^{-\lambda} \cdot \sum_{\bar{I}_{i} \subset I_{i}, i \in B} l\left(I_{B}\right)^{p(\rho-n)+\lambda} \cdot \mu(I)^{p}$

by the second case applied to the measure $\mu$. Combining the last two inequalities with the definition of $W_{\rho, \lambda, p}^{d y} \mu$ and summing over all choices of $A$ and $B$ proves (6) also in this last case.

\section{References}

[1] D. R. Adams, L-I. Hedberg, Function Spaces and Potential Theory, Springer Verlag, Berlin, 1995 
[2] D. R. Adams, Jie Xiao, Nonlinear Potential Analysis on Morrey Spaces and Their Capacities, Indiana Math. J., 53(2004)6, pp. 1629 - 1663

[3] S. Campanato, Proprietà di inclusione per spaze di Morrey, Ricerche Mat. 12(1963), pp. $67-86$

[4] L-I. Hedberg, Th. Wolff, Thin sets in nonlinear potential theory, Ann. Inst. Fourier, Grenoble, 33(1983)4, pp. $161-187$

[5] C. B. Morrey Jr, On the solution of quasi-linear partial differential equations, Trans. Amer. Math. Soc. 43(1938), 126 - 166

[6] D. K. Palagachev, L. G. Softova, Singular Integral Operators, Morrey Spaces and Fine Regularity of Solutions of PDE's, Potential Analysis, 20(2004), pp. $237-263$

[7] J. Peetre, On the theory of $\mathcal{L}_{p, \lambda}$ spaces, J. of Functional Analysis, $4(1969)$, pp. $71-87$

[8] T. Sjödin, Thinness in non-linear potential theory for non-isotropic Sobolev spaces, Ann. Sci. Acad. Fenn., 22(1997), pp. 313 - 338

[9] G. Stampaccia, The spaces $\mathcal{L}^{(p, \lambda)}, N^{(p, \lambda)}$ and interpolation, Ann. Scuola Norm. Sup. Pisa, 19(1965), pp. $443-462$

[10] B. O. Turesson, Nonlinear Potential Theory and Weighted Sobolev Spaces, Lecture Notes in Mathematics, No 1736, Springer Verlag, Berlin, 2000

Address: Department of Mathematics and Mathematical Statistics, University of Umeå, S-901 87 Umeå, Sweden. E-mail: tord.sjodin@math.umu.se 